\title{
From Effluent to New Water: Performance Evaluation and Quality Assurance
}

\author{
Martin Reinhard*, John Montgomery-Brown, Jennifer S. Louie, and Birgit Gross
}

\begin{abstract}
As water reuse becomes increasingly important to satisfy water demand, ensuring the quality of recycled wastewater becomes ever more vital. Pharmaceuticals (PhACs) and alkylphenol polyethoxylates (APEOs) metabolites are two groups of chemicals that are commonly present in treated effluent and have received attention for their demonstrated or potential biological effects. In this paper we present data on the effects of river transport, wetland treatment, and groundwater recharge on the attenuation of these emerging chemicals. Using data from three advanced water treatment plants, we also report on the efficiency of microfiltration, reverse osmosis and ultraviolet oxidation in removing these compounds from advanced treated effluents. With respect to natural attenuation processes, decreases in pharmaceutical concentrations during river transport were likely attributed to sediment sorption and chemical and biological degradation or transformation. Wetland treatment was less efficient when compared to river transport. Groundwater recharge appeared to be an effective removal process (> 99\% attenuation) for PhACs and APEO metabolites, although trace levels of the latter can travel substantial distances in the subsurface. With regards to the engineered treatment options, reverse osmosis was capable of almost complete rejection of all PhACs and APEO metabolites analyzed, whereas the performances of microfiltration and UV treatment were much less efficient and consistent.
\end{abstract}

Keywords: Alkylphenol polyethoxylate metabolites · Groundwater recharge · Pharmaceuticals · Reverse osmosis - Water reuse

\section{Introduction}

As water shortages are growing in severity worldwide, wastewater is being more seriously considered as a valuable resource. Today, increasing fractions of wastewater are being recycled for a range of applications, from irrigation and recreation to potable use and medical processes requiring ultrapure water. The presence of a variety of organic micropollutants such as pesticides and priority pollutants in advanced treated effluent has long been of interest and has raised issues about the quality of recycled water [1]. Concerns regarding these

${ }^{\star}$ Correspondence: Prof. M. Reinhard Department of Civil and Environmental Engineering Stanford University

Stanford, CA 94305-4020, USA

Tel.: +1-650-723-0308

Fax: +1-650-723-7058

E-Mail: reinhard@stanford.edu pollutants have generally been alleviated by implementing appropriate treatment methods to achieve regulatory water quality standards. In particular, the combination of microfiltration (MF), reverse osmosis (RO) and ultraviolet (UV) oxidation is increasingly being used to produce high quality water from biologically treated effluent. However, the data available are still insufficient to predict the potential for contaminants to pass through RO membranes and to understand the properties that control compound rejection. Natural treatment processes such as river transport, wetland treatment, and groundwater recharge can improve water quality in intentional and incidental (i.e. river-groundwater) water reuse applications. The natural attenuation processes that occur in these systems are poorly documented and therefore it is difficult to consider them as water purification processes in water recycling schemes, although notable exceptions exist [2]. The ability and efficiency of natural processes and treatment technologies to remove trace levels of impurities are of utmost impor- tance if we are to rely on water recycling as a standard practice in the future.

Water quality research interests have recently turned towards groups of contaminants that demonstrate biological effects but are not all currently subject to regulatory water quality limits. Two groups that belong in this category are endocrine disrupting chemicals (EDCs) and pharmaceutically active compounds (PhACs). Researchers have shown that some EDCs can interfere with the reproduction and development of humans and other animals, even at extremely low concentrations (i.e. on the order of nanograms per liter and lower) [3]. Examples of EDCs that elicit these effects are metabolites of alkylphenol polyethoxylates (APEOs), nonionic surfactants that are found in industrial and household cleaning products and are also used in a variety of industries (e.g. plastics, textiles, and paper manufacturing). These metabolites - including alkylphenols (APs), alkylphenol ethoxycarboxylates (APECs) and carboxylated alkylphenol ethoxycarboxylates (CAPECs) - can be produced via biotrans- 
formation of APEOs during sewage or wastewater treatment [4]. According to a survey conducted by United States Geological Survey [5], APEOs and their metabolites are among the most commonly detected wastewater-derived contaminants in US surface waters. These compounds have also been detected in advanced treated wastewater effluents at levels commonly in the nanograms or low micrograms per liter range [4]. Most of the environmental data has been obtained for APEOs, whereas APECs and especially CAPECs have received much less attention.

Although the occurrence of PhACs in surface water and advanced treated effluents has been widely reported [6-8], our knowledge of their environmental significance is incomplete. Since PhACs are not completely degraded in humans before being excreted, they can be found in domestic wastewaters in their active forms along with their metabolites [6]. PhACs in wastewater may undergo transformations during treatment, but analyses of effluent treated by activated sludge, tertiary filtration and microfiltration have shown that these compounds and their metabolites are not completely removed [7]. Although $\mathrm{PhACs}$ have been detected in the environment at very low levels (i.e. at concentrations generally less than $1 \mu \mathrm{g} / \mathrm{l})$, there are concerns that they may act synergistically and produce chronic effects if they accumulate in higher organisms [6][9-10].

Because the potential or demonstrated biological effects of water containing trace levels of PhACs and EDCs could impact the quality of recycled water and provide the impetus for new regulatory water quality standards, it is important to understand how these chemicals attenuate in surface and subsurface waters, as well as how they can be completely removed from wastewater effluents. This paper presents and evaluates recent data developed in this laboratory related to the natural attenuation of these emerging contaminants during river transport, wetland treatment, and groundwater recharge. An evaluation of the removal efficiencies for these impurities in three water treatment systems involving microfiltration (MF), reverse osmosis (RO) and UV oxidation is also presented.

This study focused on various neutral and carboxylated APEO metabolites, as well as PhACs including carbamazepine, gemfibrozil, ibuprofen, ketoprofen, and naproxen. Because these emerging compounds include diverse groups of chemicals, may have single or multiple acidic functional groups, and are potentially environmentally significant at the nanograms per liter level, more sophisticated analytical methods were developed for their detection methods to a broader range of treatment systems and more detailed cases studies will help to improve treatment procedures and ensure the quality of water supplies that contain large fractions of effluent.

\section{Analytical Methods}

The procedure used was based on solidphase extraction (SPE) to concentrate the contaminants of interest, as indicated in Fig. 1 and described in more detail elsewhere [11]. After allowing water samples (1-8 liters) to equilibrate to room temperature, the $\mathrm{pH}$ was adjusted to 2 using sulfuric acid. The $\mathrm{C}-18$ SPE cartridges $(1 \mathrm{~g})$ were tone, $5 \mathrm{ml}$ of acetonitrile and $5 \mathrm{ml}$ of distilled deionized water ( $\mathrm{pH} 2$ ). Water samples were extracted at a rate of $15 \mathrm{ml} / \mathrm{min}$ or less. Following extraction, the cartridges were dried for approximately $2 \mathrm{~h}$ under a gentle vacuum and extracted using acetone and acetonitrile. Extracts were concentrated to approximately $30 \mu \mathrm{l}$ under a gentle stream of nitrogen and divided into three aliquots. One aliquot was derivatized to convert carboxylic acids into propyl esters; the second aliquot was silylated using $\mathrm{N}$ (tert-butyldimethylsilyl)-N-methyl-trifluoroacetamide (MTBSTFA); and the third was analyzed without derivatization for and quantification. Application of such conditioned sequentially with $5 \mathrm{ml}$ of ace-

neutral chemicals. Target compounds were identified and quantified by GC-MS or GCMS/MS using Electron Impact (EI) ionization. APs, APEOs, and APECs, which occur as complex mixtures that are not completely separated by this analytical technique, were identified using the base peak and several qualifier ions and quantified in the full scan mode. PhACs and other EDCs were detected by monitoring several specific fragment ions and quantified using the base peak. The detection limits for this procedure ranged from 0.5 to $1.0 \mathrm{ng} / \mathrm{l}$ and the reporting limit from 1.0 to $5.0 \mathrm{ng} / \mathrm{l}$.

\section{Results and Discussion}

\section{River Transport and Wetland \\ Treatment}

During the dry season, the Santa Ana River (SAR) is predominantly fed by tertiary treated effluents at various locations along its length [12]. Because SAR water is used to replenish the groundwater basin in Orange County, California, concerns have been raised that wastewater impacts may eventually degrade this vital water supply. Water samples were collected at four locations along a wastewater-augmented section of the SAR to examine the attenuation of two PhACs, ibuprofen and gemfibrozil. Details on the attenuation of other compounds in the SAR are reported elsewhere [11].

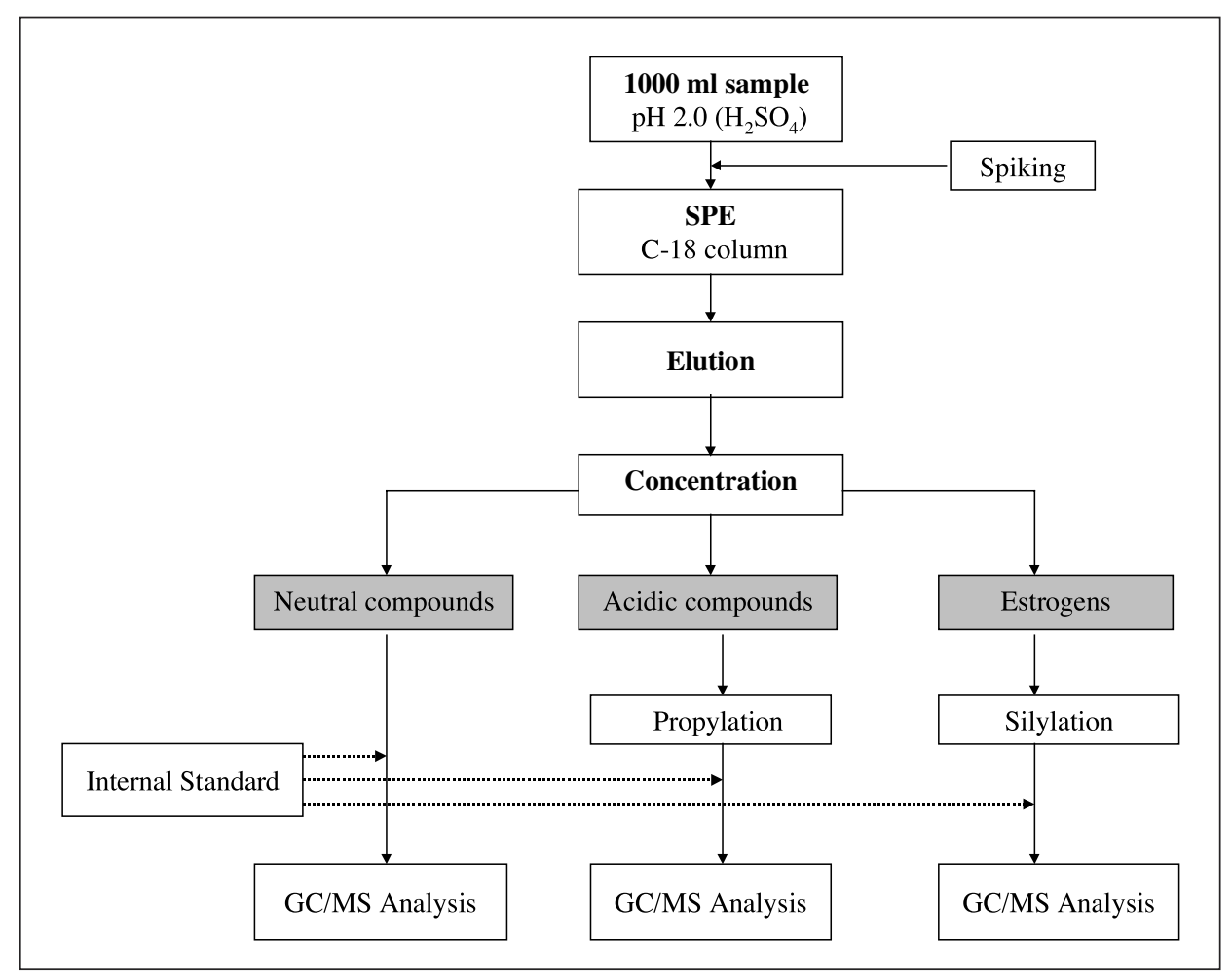

Fig. 1. Schematic of analytical approach. 
Fig. 2a shows schematically the river sampling locations (SAR-1 to SAR-4), the treated effluent discharge points, the wetland inflow and outflow locations, and the recharge area in Orange County. Fig. $2 \mathrm{~b}$ and $2 \mathrm{c}$ show the concentrations at four sites of ibuprofen and gemfibrozil, respectively, as well as the concentrations of these PhACs in the treated effluent inputs. The composition of river water at SAR-1 is almost $100 \%$ treated effluent. Both PhACs were detected in all four treated effluents, and both $\mathrm{PhAC}$ concentrations at SAR-4 were lower than the corresponding concentrations at SAR-1.

The impact of river transport on the $\mathrm{PhAC}$ levels in the absence of wastewater treatment plant inputs is illustrated by the SAR-2 to SAR-3 interval. During the approximately seven-hour period of travel between these two points, the concentrations of ibuprofen and gemfibrozil rapidly decreased by $88 \%$ and $76 \%$, respectively. These significant decreases were also observed for the other compounds [11], and are believed to be the result of sediment sorption, although the roles of this and other environmental processes (e.g. photo-, chemical or biological degradation) cannot be elucidated without additional data.

Despite the longer retention time involved with wetland treatment (which had

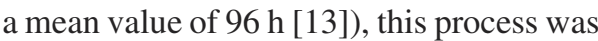
less effective than river transport at reducing ibuprofen concentrations (from $17 \mathrm{ng} / \mathrm{l}$ to $9 \mathrm{ng} / \mathrm{l}$, or $54 \%$ removal), and was ineffective at reducing gemfibrozil levels. However, because of the limited sampling data, low target compounds concentrations and high variability of the wetland influent, conclusions on the effect of wetland treatment should not be drawn.

\section{Groundwater Recharge}

Groundwater recharge using wastewater effluents is increasingly being used in arid environments to help replenish groundwater supplies. However, there is concern about whether any chemicals present in wastewater effluents persist long enough to impact drinking water supplies. To address this concern, a groundwater recharge facility in Mesa, Arizona has been monitoring trace organic concentrations in downgradient wells since 1997 . The capacity and operating conditions of this facility can be found elsewhere [14-16].

Fig. 3 illustrates the distributions and average concentrations (between 2002 and 2003) of carboxylated APEO metabolites (i.e. APECs and CAPECs) in the Mesa tertiary effluent and three downgradient wells (NW4, NW2, and 26U, which are located approximately 390, 890, and $2000 \mathrm{~m}$, re-

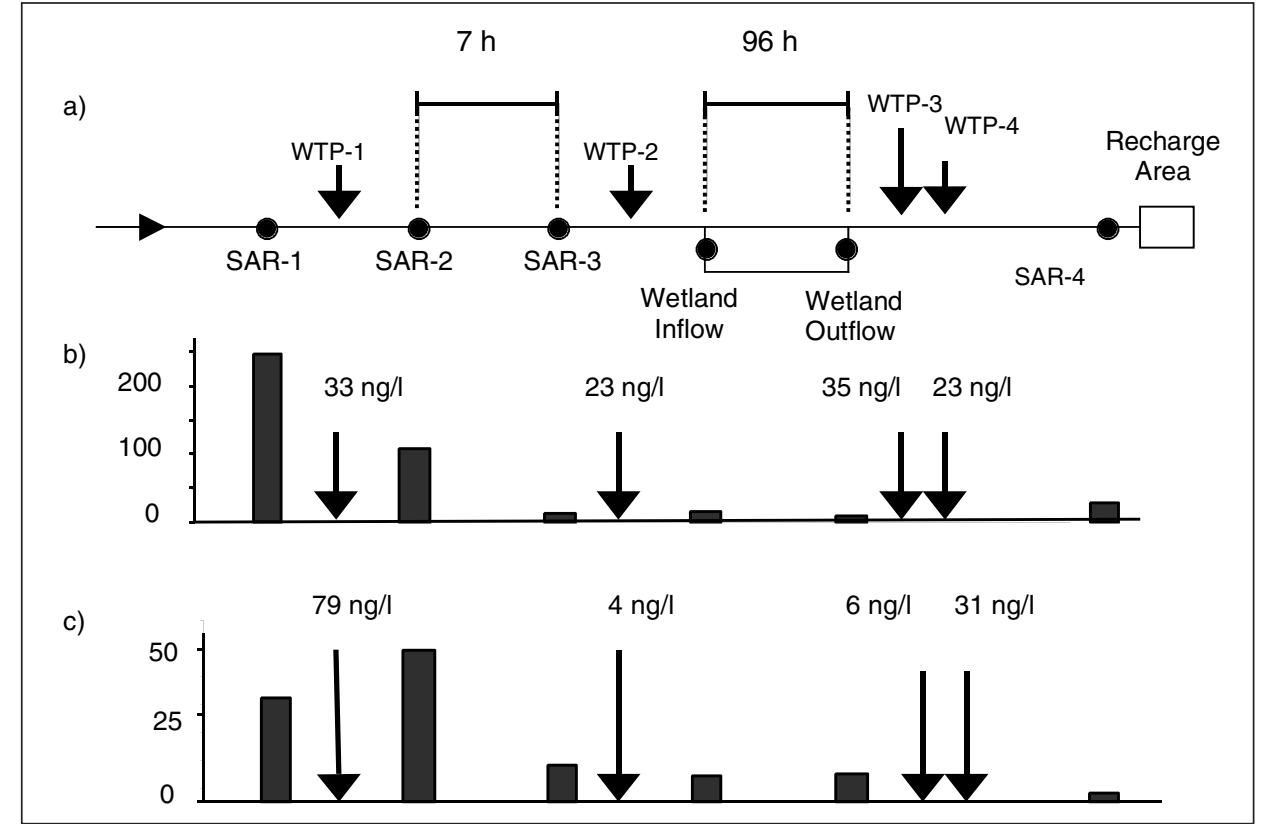

Fig. 2. a) Schematic of the SAR flow showing the four river sampling points (SAR-1 etc., shown as circles along the $x$-axis), wetland in- and outflow (shown below the $x$-axis as circles), and the four different treatment plant inputs (WTP-1 etc., shown as arrows) into the river. b) Concentration distribution of the PhAC ibuprofen along the river pathway during the September 2002 sampling round. c) Concentration distribution of the PhAC gemfibrozil along the river pathway during the September 2002 sampling round.

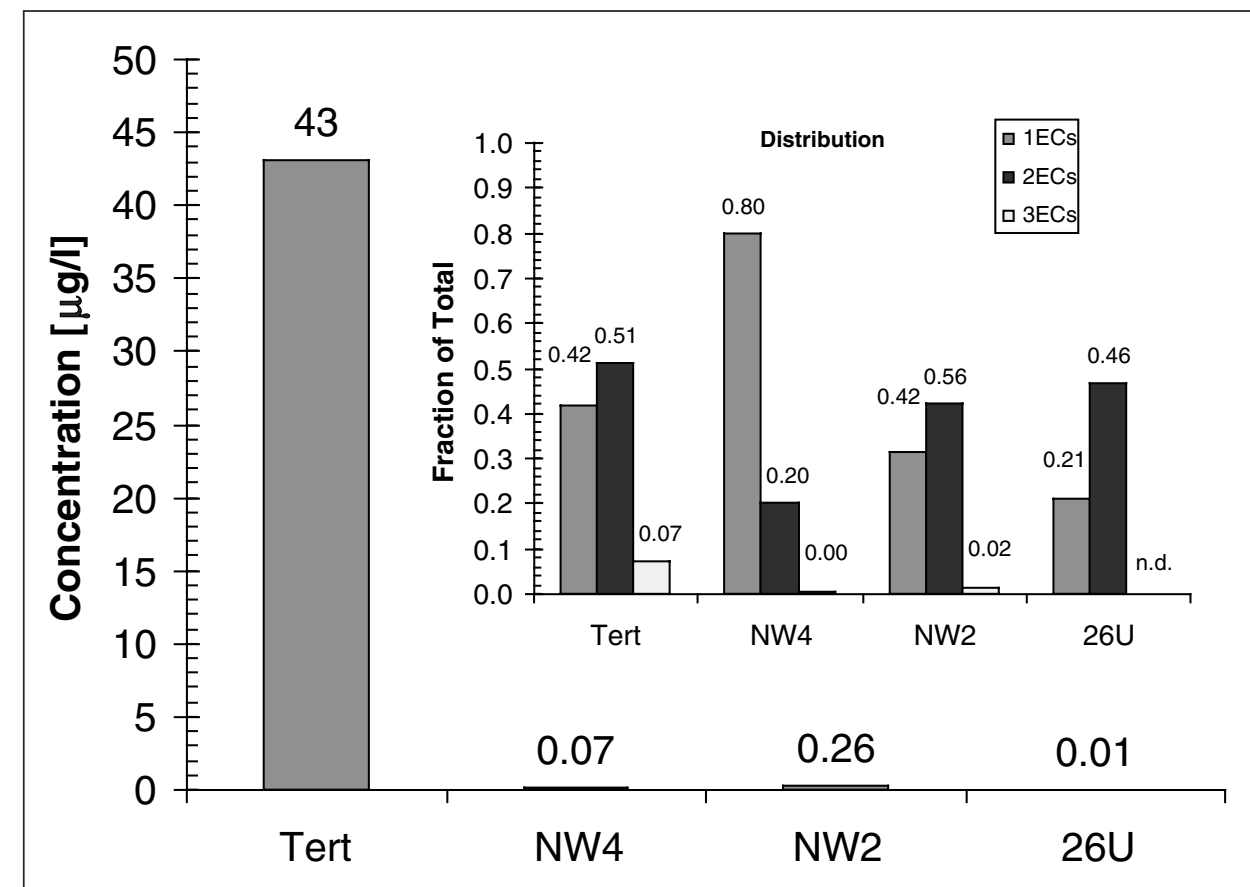

Fig. 3. Average concentrations of carboxylated APEO metabolites as a function of distance from the Mesa NWWRP recharge basins. Tert - Tertiary effluent. NW2, NW4, and $26 \mathrm{U}$ - downgradient wells. nECs - no. of ethoxy units present in the APEO metabolite. 
spectively, from the recharge basins) between 2002 and 2003. These results are in line with the average concentrations observed since 1997 [17]. As can be seen, the concentration of carboxylated APEO metabolites decreases to trace levels between the recharge basins and NW4; concentrations thereafter remain essentially constant. Of the metabolites detected, those with one or two ethoxy groups (the 1ECs and 2ECs) appear to be the most persistent. A limitation to these results is the lack of resolution between the recharge basins and NW4 (more detailed information on the attenuation of these compounds within the first three meters of the ground surface is presented in [16]); however, they do highlight two questions: Are these metabolites APECs or CAPECs, and why do low concentrations of carboxylated APEO metabolites persist? The first question is important with respect to determining the environmental significance of these compounds (e.g. CAPECs are likely much less estrogenic than APECs and APs) and the second is important for understanding the dominant processes involved in the attenuation of carboxylated APEO metabolites in the environment and the limitations of groundwater recharge.

Fig. 4 shows the distribution and the total concentration of pharmaceutical compounds in the Mesa tertiary effluent between 2002 and 2003. Of the metabolites detected, gemfibrozil and an ibuprofen metabolite were detected in all samples and naproxen and ibuprofen were detected in three samples. It is unknown why the concentrations of pharmaceuticals in the tertiary effluent were so much higher in October 2002 and January 2003, than they were in January and April 2002; the carboxylated APEO metabolites exhibited the same trend. No pharmaceuticals were detected in the any of the downgradient wells; however, more data are needed to ascertain that pharmaceuticals are removed during groundwater recharge.

\section{Reverse Osmosis Treatment}

Water quality data from three water treatment systems were collected to investigate the ability of $\mathrm{RO}$ membranes to reject PhACs and APEO metabolites from advanced treated effluents (Table). The three sites vary in size and combination of treatment steps; however, all three use polyamide RO membranes. Sites 1 and 2 are full-scale water treatment plants that employ RO and UV, with MF preceding RO treatment at Site 1 . Site 3 is a pilot-scale system that employs MF and RO. Since effluent samples were collected from various points within each treatment system, the re-

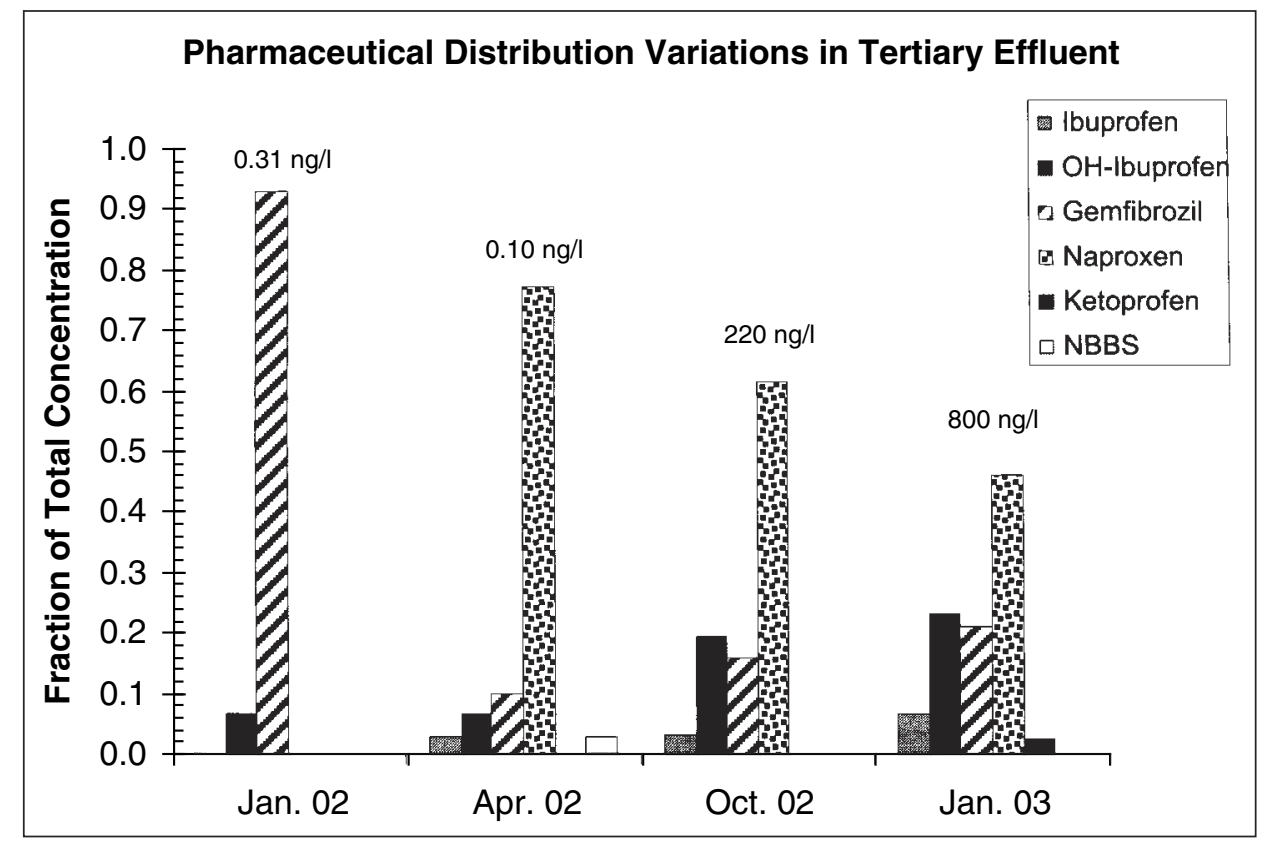

Fig. 4. Pharmaceutical distribution and concentrations in the Mesa tertiary effluent. Total concentration of PhACs is displayed above bars. The order of individual PhACs in legend reflects the order displayed in chart from left to right.

jection capabilities of the treatment technologies can be compared.

Data from Sites 2 and 3 indicate that microfiltration does not consistently or effectively reject the selected suite of organic compounds. At Site 2, the rejection percentages were $12.5 \%$ for carboxylated APEO metabolites (which refers to both APECs and CAPECs), 33\% for ibuprofen (the only pharmaceutical detected) and $45 \%$ to $74 \%$ for neutral APEO metabolites (neutrals). Despite the complete rejection of some neutrals at Site 3, microfiltration failed to effectively remove carboxylated APEO metabolites (74\% rejection) or pharmaceuticals (ranging from $15 \%$ for gemfibrozil up to $80 \%$ for naproxen). The apparent complete removal of NBBS may be an artifact of its low concentration in the influent water.

In contrast to microfiltration, reverse osmosis with polyamide membranes at Sites 1, 2, and 3 was capable of consistently achieving almost $100 \%$ rejection of all neutrals, carboxylated APEO metabolites and pharmaceuticals analyzed. This finding is consistent with the RO rejection data presented by Reinhard et al. [18] for one full-scale and two pilot-scale advanced water treatment systems (Table). The two pilot plant systems were equipped with polyamide membranes and exhibited similar rejection efficiencies for neutrals (95-98\%) and carboxylated APEO metabolites (96-99\%). As shown in the Table, the cellulose acetate membrane used in the fullscale plant was consistently less effective at rejecting trace organics relative to the polyamide membranes. Although the rejec- tion ranges for the polyamide membranes used in the 1986 study [18] are very high they are slightly lower than the near complete removals observed at Sites 1,2 and 3, which suggests that polyamide membrane rejection capabilities have improved over the last 15 years. foreshadowed the efficient rejection of select pharmaceuticals by polyamide membranes, with the almost complete rejection of clofibric acid (a PhAC) by both pilotscale systems. The near complete rejection of pharmaceuticals has also been reported by others [7] at a full-scale water treatment plant in Scottsdale, Arizona. As shown in the Table, none of the PhACs evaluated in the Scottsdale study was detected in the effluent after treatment by microfiltration and reverse osmosis using polyamide membranes.

The rejection of organic compounds by RO membranes has been studied extensively [19-22] and is influenced by the physical and chemical properties of the rejected molecules and of the membrane itself. For the chemicals included in this study, steric hindrance likely plays a role in the rejection of both the neutral and charged compounds due to their large (and in some cases branched) structures [19]. Sorption of organics to the hydrophobic parts of the RO membrane is another possible retention mechanism [22]. Charge repulsion is another factor that influences the rejection of acidic compounds, such as carboxylated APEO metabolites and some pharmaceuticals. A large fraction of these molecules are negatively charged at $\mathrm{pH}$ levels above their
The results from the 1986 investigation 
Table. Summary of removal data for APEOs, APECS and pharmaceuticals by seven advanced water treatment systems.

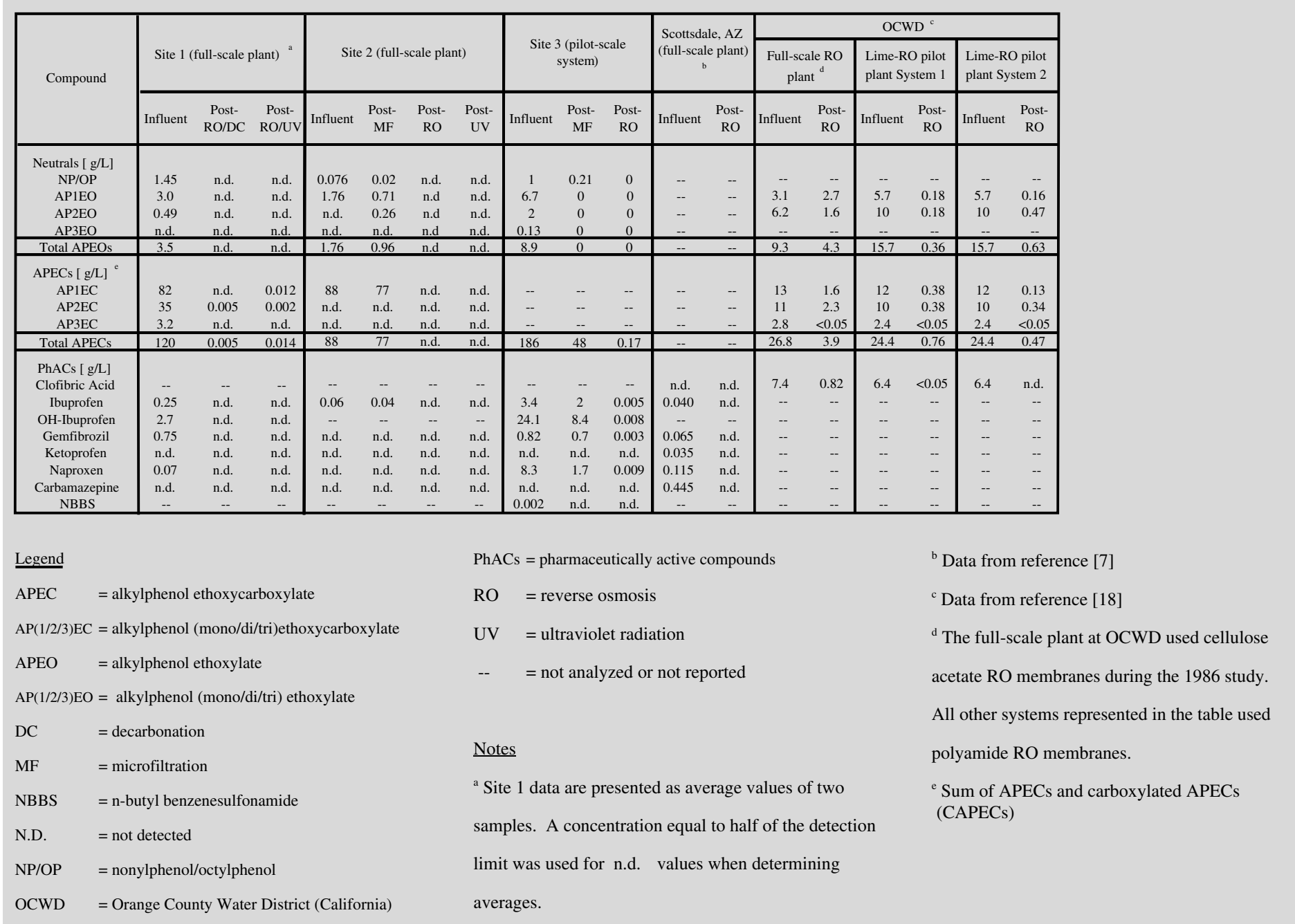

respective pKa values and thus will be repelled by the polyamide membrane surface, which is negatively charged above $\mathrm{pH} 5$ [21]. Given that the $\mathrm{pH}$ for the three sites monitored for this study was generally between 6 and 7, it is likely that significant fractions of the acidic compounds were present in their dissociated, anionic states.

Based on data from Sites 1 and 2, the efficacy of UV treatment in removing trace organics is unclear. The complete rejection of all tested compounds by RO treatment at Site 2 precluded an evaluation of UV treatment for that system. However, Site 1 data suggest that UV may not be an effective way to remove residual carboxylated APEO metabolites following RO treatment. Metabolites having one or two ethoxy units (AP1EC and AP2EC) were detected in UVtreated effluent at 0.004 and $0.012 \mu \mathrm{g} / \mathrm{l}$, respectively. Due to the relatively low concentrations of carboxylated APEO metabo- lites detected, additional experimentation is required to determine if UV oxidation can effectively treat trace levels of organics.

\section{Conclusions}

Several studies have been conducted to ascertain the removal of organic residuals from wastewater effluents that are intended for potable or non-potable reuse. The set of organic contaminants studied included PhACs and a number of wastewater indicator compounds called alkylphenol polyethoxylate (APEO) metabolites. Carboxylated APEO metabolites were the predominant contaminant class in effluents, and were present consistently at concentrations higher than $1 \mu \mathrm{g} / \mathrm{l}$. Natural attenuation of of two PhACs (ibuprofen and gemfibrozil) was found to be effective; after river and wetland flow, the concentrations of the tar- get compounds at the location where water is captured for recharge (SAR-4) were lower than the levels in secondary (activated sludge) and tertiary (advanced) treated effluents. Sorption of the organic compounds is thought to be an important removal mechanism, although other processes such as photo-, chemical- and biological degradation or transformation may also be factors. Additional data is required to understand the methods of river transport attenuation for these two chemicals. Wetland treatment over an average residence time of $96 \mathrm{~h}$ produced a smaller decrease in ibuprofen concentrations (54\%) than river transport $(88 \%)$, and did not lead to any decrease in gemfibrozil concentrations. Groundwater recharge was found to be an effective removal process. In water with several months of residence time, PhACs were not detected and carboxylated APEO metabolites were detected only at trace levels. 
With respect to the engineered treatment options evaluated, microfiltration did not consistently or effectively reject the selected suite of organic compounds. However, reverse osmosis using polyamide membranes was capable of consistently achieving almost $100 \%$ rejection of all target compounds. Results were inconclusive with respect to the elimination of trace organics using UV oxidation. Finally, it should be noted that the results presented herein applies only to the specific sites described, and should not be extrapolated without further verification of the data.

\section{Acknowledgements}

This work was supported in part by the STC program of the National Science Foundation under Agreement Number CTS-0120978, by the United States Environmental Protection Agency (US EPA), and the American Water Works Association Research Foundation (AWWARF), through grants to the National Center for Sustainable Water Supply (Arizona State University, University of Arizona, University of Colorado, Stanford University); and by the Orange County Water District, Fountain Valley, California.

Received: July 26, 2003
[13] A.Y. Lin, J.-F. Debroux, J.A. Cunningham, M. Reinhard, Ecolog. Eng. 2003, 20, 75-88.

[14] J. Drewes, P. Fox, Water Sci. Tech. 1999, 40(4-5), 391-398.

[15] P. Fox, K. Narayanaswamy, A. Genz, J. E. Drewes, Water Sci. Tech. 2001, 43(10), 343-350.

[16] J. Montgomery-Brown, J.E. Drewes, P. Fox, M. Reinhard, Water Res. 2003, 37(15), 3672-3681.

[17] P. Fox, S. Houston, P. Westerhoff, J. Drewes, M. Nellor, W. Yanko, R. Baird, M. Rincon, R. Arnold, K. Lansey, R. Bassett, C. Gerba, M. Kariscak, G. Amy, M. Reinhard, 'An Investigation on SoilAquifer Treatment for Sustainable Water Reuse,' Am. Water Works Assoc. Research Foundation and the Am. Water Works Assoc., Denver, CO, 2001, p. 741.

[18] M. Reinhard, N.L. Goodman, P.L. McCarty, D.G. Argo, J. Am. Water Works Assoc. 1986, 78(4), 163-174.

[19] W.A. Duvel, T. Helfgott, J. Water Poll. Cont. Fed. 1975, 47(1), 57-65.

[20] L. Kastelan-Kunst, K. Kosutic, V. Dananic, B. Kunst, Water Res. 1997, 31(11), 2878-2884.

[21] H. Ozaki, H. Li, Water Res. 2002, 36(1), 123-130.

[22] L.D. Nghiem, A.I. Schafer, T.D. Waite, Water Sci. Tech. 2002, 46(4), 265-272.

[1] R.C. Cooper, Water Sci. Tech. 1991, 24(9), 55-65.

[2] C. Tixier, H.P. Singer, S. Oellers, S.R. Muller, Environ. Sci. Tech. 2003, 37(6), 1061-1068.

[3] S. Jobling, D. Sheahan, J.A. Osborne, P. Mat-Thiessen, J.P. Sumpter, Environ. Toxicol. Chem. 1996, 15(2), 194-202.

[4] J. Montgomery-Brown, M. Reinhard, Environ. Eng. Sci. 2003, 20(5), 471-486.

[5] D.W. Kolpin, E.T. Furlong, M.T. Meyer, E.M. Thurman, S.D. Zaugg, L.B. Barber, H.T. Buxton, Environ. Sci. Tech. 2002, 36(6), 1202-1211.

[6] O.A.H. Jones, N. Voulvoulus, J.N. Lester, Environ. Tech. 2001, 22(12), 1383-1394.

[7] J.E. Drewes, T. Heberer, K. Reddersen, Water Sci. Tech. 2002, 46(3), 73-80.

[8] T.A. Ternes, M. Meisenheimer, D. McDowell, F. Sacher, H.J. Brauch, B. HaistGulde, G. Preuss, U. Wilme, N. ZuleiSeibert, Environ. Sci. Tech. 2002, 36(17), 3855-3863.

[9] E. J. Routledge, D. Sheahan, C. Desbrow, G. C. Brighty, M. Waldock, J.P. Sumpter, Environ. Sci. Tech. 1998, 32(11), 1559-1565.

[10] E. Silva, N. Rajapakse, A. Kortenkamp, Environ. Sci. Tech. 2002, 36(8), 1751-1756.

[11] B. Gross, M. Reinhard, 'Occurrence and Fate of Emerging Contaminants in the Santa Ana River and Prado Wetland, Final Report', submitted to Orange County Water District, 2003.

[12] W.-H. Ding, J. Wu, M. Semadeni, M. Reinhard, Chemosphere 1994, 39(11), 1781-1794. 